\title{
Analyse des bruits émis par les valves cardiaques artificielles
}

\author{
C. MASSON et P.J.T. FILIPPI*
}

Institut de Mécanique des Fluides de Marseille, 1 rue Honnorat, 13003 Marseille, France

${ }^{*}$ Laboratoire de Mécanique et d'Acoustique, 31 chemin Joseph Aiguier, 13402 Marseille cedex 20, France

\begin{abstract}
The aim of this study is to compare the noise emitted by mechanical artificial valves to that produced by biological ones which are not disturbing for the patients. We use an in vitro approach. The valves, in the mitral position, are placed on a cardiovascular similator composed of an elastic atrio-ventricular prosthesis and an hydrodynamic generator. This assembly generates a periodic pulsatile flow. The noise signal is recorded with a hydrophone located in the excitation tank, close to the valve. Three different prostheses have been investigated : ball, tilting disk, bileaflet valves.

During each cardiac cycle, the recorded signal is composed of a short burst of large amplitude which corresponds to the valve closure, and then a low level component at much lower frequency. To analyse such a burst, a time-frequency technique is much more convenient than a classical Fourier analysis. The wavelet analysis method appeared to be very efficient : it points out the time fluctuations of each frequency component.

The results obtained show the efficiency of this technique. As far as the results are concerned, it appears that mechanical valves produce a high level sound within the octave bandwidth centered at $64 \mathrm{~Hz}$ up to $512 \mathrm{~Hz}$, while the amplitude of these components decreases rapidly for the biological valve. The sound level difference is about $20 \mathrm{~dB}$.
\end{abstract}

\section{INTRODUCTION}

En 1983, une enquête clinique menée sur des patients ayant une prothèse valvulaire mécanique a montré que la moitié d'entre-eux étaient perturbés par le bruit émis par leur prothèse et un tiers d'entre-eux ont rapportés que leurs proches l'étaient également[1]. Une étude plus récente (1990-1991)[2] a donné des résultats similaires. De plus, d'un point de vue médical, la surveillance post-opératoire est essentielle. A cause du risque couru par le patient par dysfonctionnement de la prothèse, des examens périodiques doivent être effectués. Ils doivent être non invasifs, à relativement faible coût, facilement répétitifs et sans risque. Dans ce but et afin de contribuer au confort du patient, nous nous sommes intéressés à l'évaluation du bruit de fermeture des valves cardiaques.

La présente étude a pour objectif de mettre au point une technique d'évaluation du bruit de fermeture des différents types de prothèses valvulaires et de développer une méthode d'analyse du bruit de fermeture pour en connaître son origine physique.

\section{METHODE EXPERIMENTALE}

La première étape de notre travail a été effectuée à l'Institut de Mécanique des Fluides de Marseille. Elle a consisté à enregistrer in vitro les bruits émis par différentes prothèses valvulaires placées en position mitrale sur le coeur gauche.

\subsection{Valves étudiées}

L'étude expérimentale que nous avons réalisée concerne, pour les valves mécaniques, une valve à bille Starr-Edwards (23 $\mathrm{mm}$ de diamètre interne), une valve à disque Björk-Shiley (22 $\mathrm{mm}$ de diamètre interne) 
et une valve à deux clapets St Jude (24 mm de diamètre interne). La bioprothèse étudiée est une valve Carpentier-Edwards (23 mm de diamètre interne).

\subsection{Moyens et conditions d'essais}

Le banc de simulation cardiovasculaire mis au point au laboratoire, produit des écoulements pulsés de forme quelconque. Ce simulateur est composé d'un circuit primaire et d'un circuit secondaire. Le circuit primaire correspond au circuit d'activation ventriculaire. Il simule les contractions musculaires au niveau du ventricule. Les variations de pression dans la cuve d'activation provoquent la compression et la dilatation de la poche ventriculaire. Le tout est plongé dans un bac contenant de l'eau. Le circuit secondaire est un modèle de circuit systémique. Nous avons placé dans la cuve d'activation ventriculaire un hydrophone le plus près possible de la valve mitrale $(L=3 \mathrm{~cm})$. En position aortique nous avons placé une valve en silicone. La mesure de la pression acoustique a été effectuée grâce à un capteur sous marin de petite taille. Ce transducteur couvre la gamme de fréquence comprise entre 0,1 et $200 \mathrm{kHz}$ avec une sensibilité en réception de $-211 \mathrm{~dB}$ (réf $1 \mathrm{~V} / \mathrm{Pa}$ ). A cet hydrophone est associé un préamplificateur à sortie couplée par transformateur. Afin de nous affranchir du signal de la pompe nous avons filtré les fréquences inférieures à $3 \mathrm{~Hz}$.(figure1)

La fréquence d'échantillonnage est de $40 \mathrm{kHz}$ pour les mesures de pression acoustique.

Les conditions d'essais sont représentatives des conditions classiques trouvées in vivo. La fréquence cardiaque est fixée à $70 \mathrm{Puls} / \mathrm{mn}$. Les signaux de pression aortique et ventriculaire sont physiologiques et répétitifs tout au long des essais. Le pic de pression ventriculaire a été fixé à $120 \mathrm{mmHg}$, le débit moyen à $5 \mathrm{l} / \mathrm{mn}$ ce qui correspond aux conditions normales pour un coeur sain.

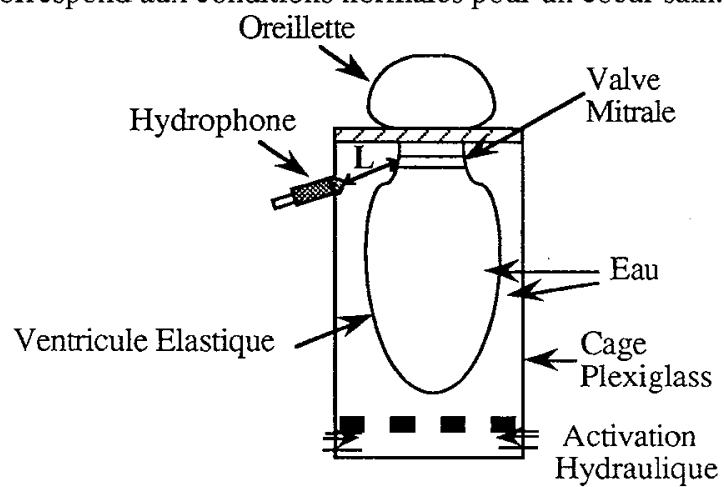

figure 1 : Cuve d'activation

\section{ANALYSE DES DONNEES}

Dans une seconde étape, nous avons analysés le bruit de fermeture des valves cardiaques. Pendant un cycle cardiaque, la partie du signal qui nous intéresse est de très brève durée (đe 15 à $25 \mathrm{~ms}$ ). De plus il s'agit d'un signal typiquement non stationnaire. Une technique temps-fréquence s'imposait. Nous avons donc adopté une analyse sur base d'ondelettes.

L'analyse en ondelettes se déroule de la façon suivante. A partir d'une seule ondelette, l'ondelette mère ou analysante $\psi_{00}(\mathrm{t})$, est engendrée une famille d'ondelettes $\psi_{\mathrm{j}, \mathrm{k}}(\mathrm{t})$. Le but consiste à écrire n'importe quelle fonction $f(t)$ comme combinaison linéaire de ces ondelettes :

$$
f(t)=\sum_{j} \sum_{k} c_{j, k} \Psi_{j, k}(t)
$$

Les $c_{j, k}$ sont appelés les coefficients d'ondelettes. Ce sont les projections de la fonction $\mathrm{f}(\mathrm{t})$ sur la base $\psi_{\mathrm{j}, \mathrm{k}}$. Ils contiennent toutes les informations spectrales et temporelles du signal. Ils s'écrivent sous la forme d'un produit scalaire :

$$
c_{j, k}=\left\langle f(t), \psi_{j, k}(t)\right\rangle \quad \Leftrightarrow \quad c_{j, k}=\int_{-\infty}^{+\infty} f(t) \psi_{j, k}^{*}(t) d t
$$

Parmi les différentes bases d'ondelettes orthogonales existantes, notre choix s'est porté sur celle proposée par P. Auscher [3]. 
Les ondelettes sont définies par

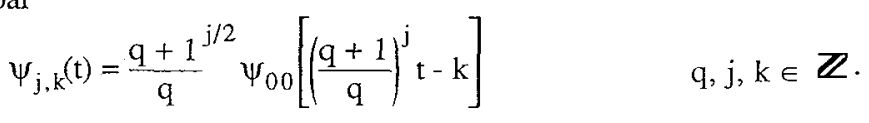

$\psi_{00}(\mathrm{t})$ est de la forme $\quad \psi_{00}(\mathrm{t})=\int_{-\infty}^{+\infty} \sin \theta_{\mathrm{q}}(v) \cos \theta_{\mathrm{q}+1}(v) \mathrm{e}^{2 \mathrm{i} \pi v \mathrm{t}} \mathrm{dv}$

où $\theta_{\mathrm{q}}$ est une fonction construite à partir de la fonction de Jaffard.

Auscher a démontré que la famille d'ondelettes $\psi_{\mathrm{j}, \mathrm{k}}$ forme une base orthogonale de $\mathrm{L}^{2}(\mathbb{R})$.

Nous avons commencé par faire une analyse par octave $(q=1)$. Nous avons fixé la fréquence centrale de l'ondelette mère à $2 \mathrm{~Hz}$ afin d'avoir des ondelettes dont le spectre est centré en $2 \mathrm{j}$ ce qui correspond pratiquement aux octaves normalisées. La plage fréquencielle balayée est $[30 \mathrm{~Hz}, 1000 \mathrm{~Hz}]$.

Afin de faciliter l'interprétation des résultats, nous avons utiliser les coefficients suivants :

$$
\gamma_{j, k}=\left(\frac{q+1}{q}\right)^{j / 2} c_{j, k}
$$

\section{RESULTATS}

Dans l'absolu, nos mesures ne sont pas représentatives de la réalité physique. Par contre, les différences existant entre les spectres de deux valves sont significatives. Elles existeront quelque soit la méthode de mesure employée car elle est liée à la paire de valves considérée.

Si l'on compare les différentes valves entre-elles, on constate que pour les octaves centrés en $32 \mathrm{~Hz}$ et 64 $\mathrm{Hz}$, les niveaux obtenus ont tous mêmes ordres de grandeur. Par contre, une différence nette apparaît entre la bioprothèse et deux types de valves mécaniques sur l'octave centrée sur $128 \mathrm{~Hz}$. Lors de la fermeture, le niveau de la valve à bille et de la valve à deux clapets est supérieur de $13 \mathrm{~dB}$ à celui de la valve biologique. Le niveau de la valve à disque se situe entre les deux.

Sur l'octave centrée sur $256 \mathrm{~Hz}$, le niveau sonore de la valve à deux clapets est le plus important. Le niveau des valve à bille et valve à disque est identique et est inférieur de $10 \mathrm{~dB}$ à celui de la valve à double ailettes. L'émission de la bioprothèse est négligeable par rapport aux autres valves. Sur l'octave centrée sur $512 \mathrm{~Hz}$, seules les valves à disque et à deux clapets émettent de façon significative. Sur cette bande d'analyse, on constate que la Björk-Shiley a un niveau maximum supérieur à celui de la valve Saint-Jude.

Nous avons représenté les résultats obtenus pour chaque type de valve aprés une analyse sur l'octave centrée sur $256 \mathrm{~Hz}$ (figure 2). Ces courbes représentent le niveau sonore défini par : $N=20 \log \gamma_{\mathrm{j}, \mathrm{k}}$ en fonction du temps, $\gamma_{\mathrm{j}, \mathrm{k}}$ étant les coefficients d'ondelettes définies précedemment. Sur une même figure, nous avons représenté les niveaux obtenus pour un type de valve mécanique (c'est à dire bruyante) et les niveaux obtenus pour la bioprothèse (non gênante pour le patient). A titre indicatif, nous avons également fait figurer le signal sonore de chaque valve mécanique.

\section{CONCLUSION}

Les résultats obtenus mettent trés clairement en évidence les différences d'émission sonore des prothèses testées. Si celles-ci sont peu significatives dans les basses et hautes fréquences, elles sont trés nettes dans la plage de fréquence allant de $128 \mathrm{~Hz}$ à $256 \mathrm{~Hz}$. Or cela correspond aux bandes de maximum de sensibilité de l'oreille.

Cependant, la question qui reste posée est celle de l'origine physique de cette émission sonore. Deux hypothèses sont envisageables. L'émission sonore enregistrée peut être principalement due au choc métallique entre l'élément mobile et le siège, ou bien son origine est purement hydrodynamique c'est-à-dire due à la variation trés rapide de pression dans le ventricule lors de la fermeture. Afin d'y répondre, une étude théorique est en cours.

Périodiques : [1] Schondube F., Keusen H. and Messmer B.J., Physical analysis of the Björk-Shiley prosthetic valve sound. J. Thorac. Cardiovasc. Surg. 86 (1983) 136-141.

[2] Moritz A., Noise level and perception of the closing click after heart valve replacement with St Jude medical and Björk-Shiley Monostrut protheses. Artif.Organs. 14 (1990) 373-376.

Livre : [3] Auscher P., Wavelets and their applications (Jones and Bastlett Publishers, 1992) pp 439-451 

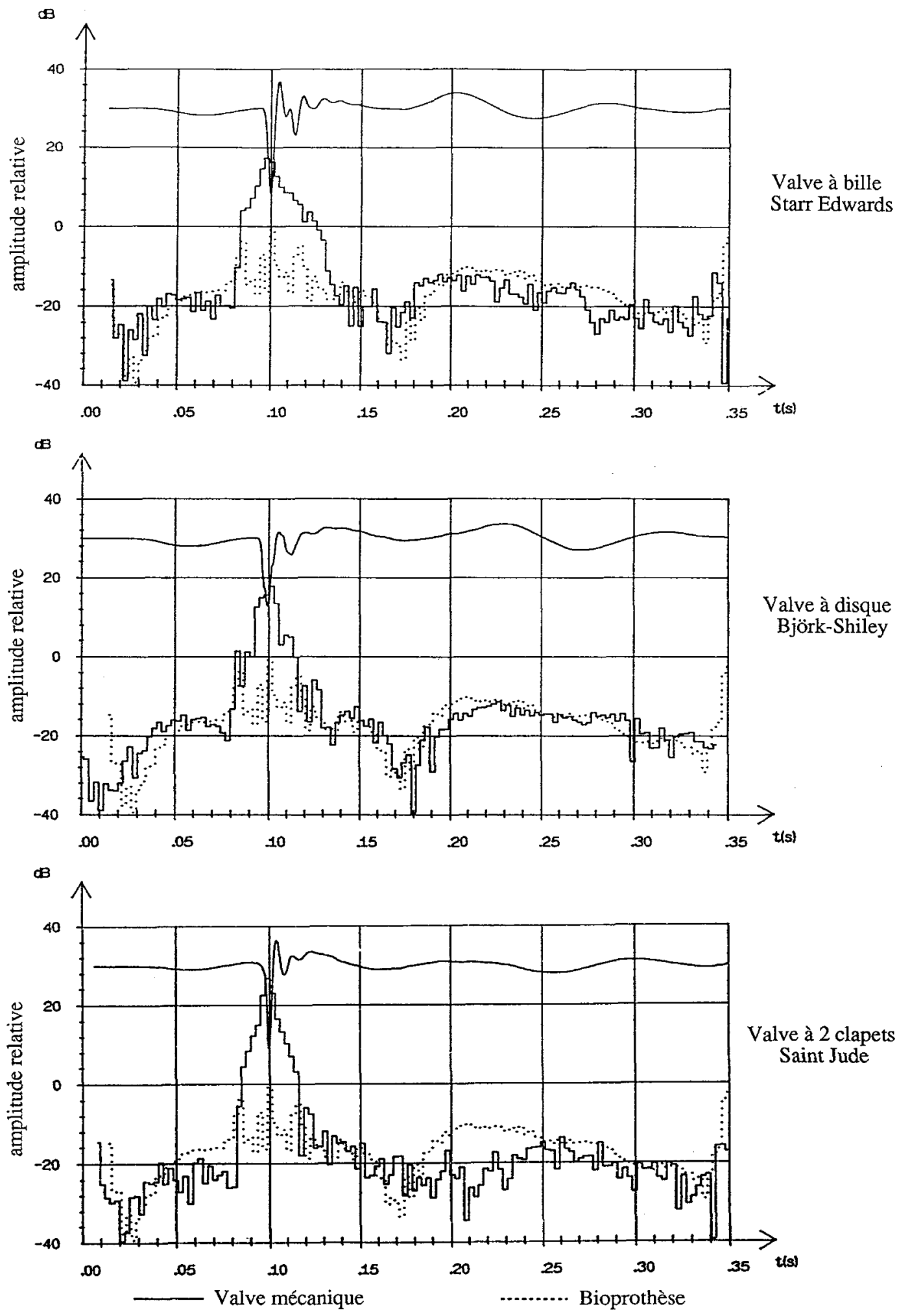

figure 2 - Niveaux : Analyse sur l'octave centrée sur $256 \mathrm{~Hz}$ 